\title{
Gallium Scan
}

National Cancer Institute

\section{Source}

National Cancer Institute. Gallium Scan. NCI Thesaurus. Code C38087.

A nuclear imaging procedure in which gallium- 67 is used as the radiopharmaceutical.

Gallium binds to transferrin, leukocyte lactoferrin, bacterial siderophores and

inflammatory proteins and thereby can be used to localize foci of infection. It is also taken up by several tumors, thus gallium scintigraphy can be used for the staging of tumors. 